Michael J. Auvdel, ${ }^{1}$ M.Sc.

\title{
Amylase Levels in Semen and Saliva Stains
}

\author{
REFERENCE: Auvdel, M. J., "Amylase Levels in Semen and Saliva Stains," Journal of Forensic \\ Sciences, JFSCA, Vol. 31. No. 2, April 1986. pp. 426-431.
}

\begin{abstract}
Amylase levels were determined for 148 semen samples and 20 saliva samples as well as for their corresponding stains. The effect of aging on the detectability of amylase activity in these stains was also investigated. The Phadebas ${ }^{(\hat{\theta}}$ amylase test was used for the quantitative assay of amylase. High levels of amylase in fluid saliva resulted in high levels being detected in saliva stains. Lower levels present in most seminal fluids produce little or no detectable amounts of amylase in stains. Interpretations are made as to the possible sources of amylase activity found in stains from laboratory casework based on both the amylase concentration and the elapsed time between collection and analysis. The evidential value of the presence or absence of amylase activity in casework stains is also discussed.
\end{abstract}

KEYWORDS: pathology and biology, amylase, saliva, semen, mixed stains

The presence of high concentrations of amylase in biological stains has been used in forensic science casework as a primary determinant for the detection of saliva [1-8]. Amylase has been chosen for this determination because, although amylase is found in other biological materials, it is rarely found in such high concentrations as it is in saliva. Yet studies by Willott of salivary amylase concentrations in stains showed that there is no level of amylase above which the presence of saliva is proven [2]. In the absence of heavy contamination by feces, human milk, or vaginal material, levels of activity above $0.02 \mu$ for a $3-$ by $3-\mathrm{mm}$ stain is unlikely to be due to anything but saliva and can be regarded as a strong indication that saliva is present $[2,3]$.

The purpose of this project was threefold. First, to determine whether high concentrations of amylase can be found in seminal fluid, and if found, to determine the frequency of occurrence. Second, to study the effects of age on the amylase levels in seminal stains made from seminal fluids that have elevated amylase activity. Third, if high levels of amylase activity can be detected in seminal stains, what possible conclusions can be drawn regarding the source of the amylase activity when dealing with stains found in laboratory casework. For the purposes of this study, any fluid semen sample with an amylase level of $200 \mathrm{U} / \mathrm{L}$ or greater was chosen for aging studies.

\section{Collection of Samples}

Semen samples were obtained from the 97th General Army Hospital in Frankfurt, West Germany. The donors were individuals undergoing pre- and post-vasectomy examinations or fertility testing. Stains were made from $150 \mu \mathrm{L}$ of seminal fluid on pieces of sterilized white cotton cloth and stored at room temperature.

Received for publication 21 May 1985; revised manuscript received 1 Aug. 1985; accepted for publication 9 Aug. 1985.

${ }^{1}$ Forensic chemist, USACIL-CONUS, Forest Park, GA. 
Saliva samples were obtained from donors in our laboratory. These samples were obtained by having the individuals spit directly into test tubes. Stains were made from $50 \mu \mathrm{L}$ of saliva on pieces of sterilized white cotton cloth and stored at room temperature.

\section{Method}

The sample size for seminal fluid was $200 \mu \mathrm{L}$. The sample size for fluid saliva was $20 \mu \mathrm{L}$. Stains for both semen and saliva consisted of 3- by 3-mm sections cut from the stained areas.

Samples were placed in centrifuge tubes, and $4 \mathrm{~mL}$ of distilled water, was added. A distilled water blank and positive controls were then prepared. The tubes were pre-incubated for $5 \mathrm{~min}$ at $37^{\circ} \mathrm{C}$ in a water bath.

One Phadebas ${ }^{\oplus}$ tablet was added to each tube, which was then immediately vorticed for $10 \mathrm{~s}$ and replaced in the water bath. Incubation at $37^{\circ} \mathrm{C}$ lasted exactly $15 \mathrm{~min}$. The reaction was stopped by adding $1 \mathrm{~mL}$ of $0.5 \mathrm{M}$ sodium hydroxide, and vorticing immediately. The resultant was either centrifuged for $5 \mathrm{~min}$ at $\geq 1500 \times g$ or filtered.

The blue supernatant was pipeted into a cuvet, after which the absorbance of the supernatant was measured at $620 \mathrm{~nm}$ against distilled water using a cuvet with a $1-\mathrm{cm}$ light path. The absorbance value of the blank was subtracted from that of the sample.

The amylase activity in International Units per litre for fluid samples can be read from a standard curve prepared under laboratory test conditions with the Phadebas test kit. The standard curve shows the units of activity per liter when measured in $0.2 \mathrm{~mL}$, and as a result, the value on the curve is 5000 times greater than that detected. Therefore, the activity for stains is taken as the number of units read from the curve divided by 5000 [2].

\section{Results}

Amylase levels found in fluid semen and saliva samples are summarized in Table 1. Amylase levels for fluid saliva were usually found to be approximately 1000 times greater than those found in fluid semen. However, one semen sample had an amylase level greater than one of the saliva samples, 6820 and $5000 \mathrm{U} / \mathrm{L}$, respectively. This high level of amylase was detected in the semen sample of a vasectomized individual whose sample still had spermatozoa present.

Figure 1 shows the frequency distribution of amylase levels found in the semen of 111 donors in which spermatozoa were present and from one donor who was aspermatic. Eight samples had levels greater than $200 \mathrm{U} / \mathrm{L}$.

Figure 2 has the frequency distribution of amylase levels found in semen from 32 donors who were vasectomized and had no spermatozoa present and 4 vasectomized donors with spermatozoa still present in their samples. Two of these samples had amylase levels greater than $200 \mathrm{U} / \mathrm{L}$.

Amylase levels found in seminal stains made from the individuals whose seminal fluids had levels of activity of $200 \mathrm{U} / \mathrm{L}$ or greater are shown in Table 2 . These levels were obtained from a

TABLE 1-Amylase activities in fluid saliva and semen samples.

\begin{tabular}{lccc}
\hline \multicolumn{1}{c}{ Body Fluid } & $\begin{array}{c}\text { No. of } \\
\text { Samples }\end{array}$ & $\begin{array}{c}\text { Range } \\
\text { (U/L) }\end{array}$ & $\begin{array}{c}\text { Average } \\
\text { (U/L) }\end{array}$ \\
\hline Saliva & 20 & $(5-108) \times 10^{3}$ & $94 \times 10^{3}$ \\
Seminal (spermatozoa) & 111 & $7-1620$ & 104 \\
Seminal (vasectomized no spermatozoa) & 32 & $1-345$ & 48 \\
Seminal (vasectomized with spermatozoa) & 4 & $20-6820$ & $1725^{\prime \prime}$ \\
Seminal (aspermatic) & 1 & 18 & $\ldots$ \\
\hline
\end{tabular}

"Excluding sample with amylase activity of $6820 \mathrm{U} / \mathrm{L}$, the average for the remaining samples would be $27 \mathrm{U} / \mathrm{L}$. 


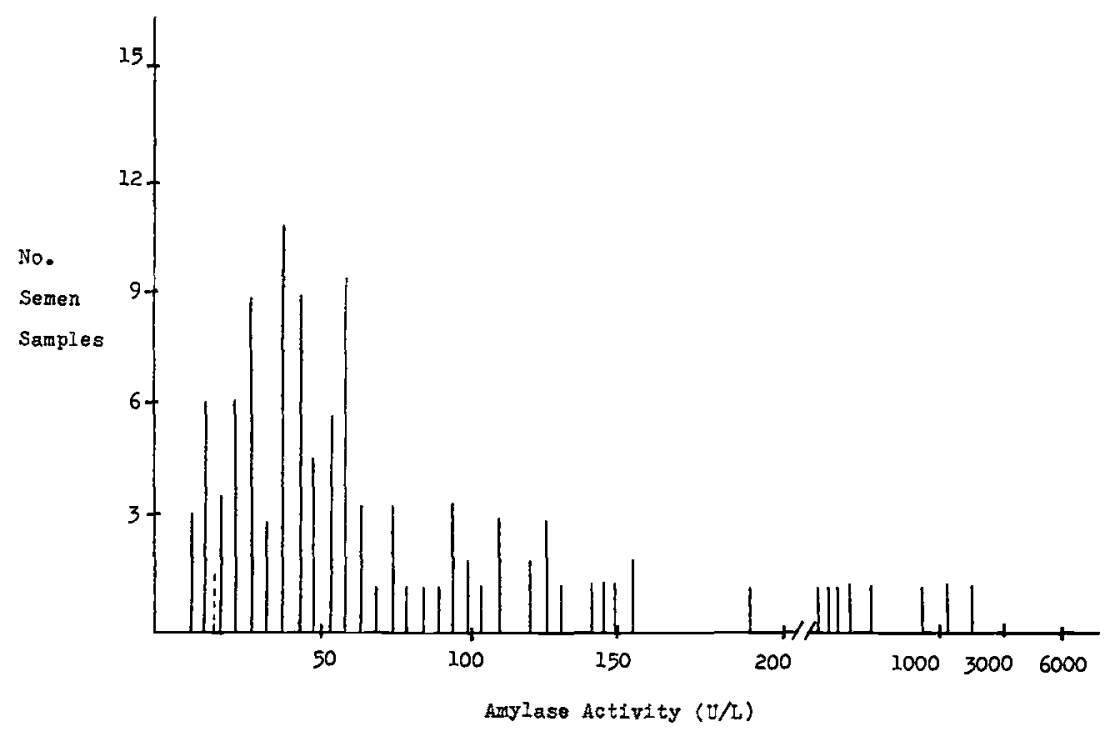

FIG. 1-Distribution of amylase activities in seminal fluids with spermatozoa present. Spermatozoa present (-) and aspermatic (---).

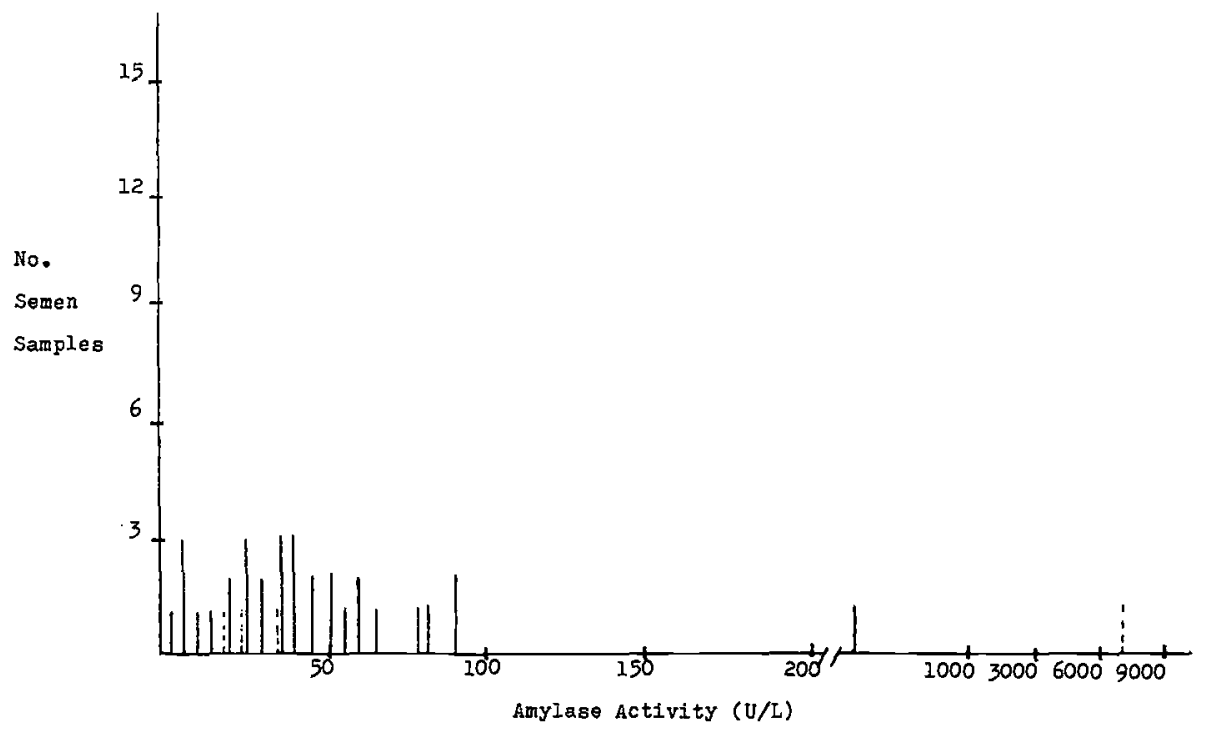

FIG. 2-Distribution of amylase activities in seminal fluids from vasectomized individuals. Vasectomy (no spermatozoa) (-) and vasectomy (spermatozoa present) (---).

3- by 3-mm section of cloth cut from a seminal stain made with $50 \mu \mathrm{L}$ of seminal fluid. Fluid samples with levels of $200 \mathrm{U} / \mathrm{L}$ or less were negative when their stains were tested. The semen sample with an original level of $6820 \mathrm{U} / \mathrm{L}$ also had a detectable amylase level in its stain. A level of 0.02 units was detectable over a two-day period. The remaining nine samples had very little amylase activity and were considered negative.

Fluid saliva and saliva stains were also tested. Saliva from 20 people and stains made from 
TABLE 2-Anylase activities in seminal stains.

\begin{tabular}{cccccc}
\hline & & \multicolumn{4}{c}{ Levels in Stains $^{b}$} \\
\cline { 3 - 6 } Sample & Fluid Level $^{a}$ & Day 1 & Day 2 & Day 5 & Day 7 \\
\hline $26^{c}$ & 6820 & 0.02 & 0.02 & 0.017 & 0.01 \\
33 & 210 & 0.001 & $\ldots$ & $\ldots$ & $\ldots$ \\
36 & 890 & 0.002 & $\ldots$ & $\ldots$ & $\ldots$ \\
56 & 1160 & 0.002 & $\ldots$ & $\ldots$ & $\ldots$ \\
75 & 400 & 0.004 & $\ldots$ & $\ldots$ & $\ldots$ \\
92 & 345 & $\ldots$ & $\ldots$ & $\ldots$ & $\ldots$ \\
104 & 265 & $\ldots$ & $\ldots$ & $\ldots$ & $\ldots$ \\
$107^{c}$ & 325 & 0.002 & $\ldots$ & $\ldots$ & $\ldots$ \\
119 & 1620 & 0.014 & $\ldots$ & $\ldots$ & $\ldots$ \\
120 & 500 & 0.003 & $\ldots$ & $\ldots$ & $\ldots$ \\
\hline
\end{tabular}

"Amylase levels in international units per litre (U/L).

${ }^{b}$ Amylase levels in international units per litre divided by $5000(\mathrm{U} / \mathrm{L} \div 5000)$.

${ }^{c}$ Vasectomized individuals.

these samples were analyzed under the same conditions as the semen samples. Amylase levels for these saliva samples are listed on Table 3 .

One sample had a low amylase level, $5000 \mathrm{U} / \mathrm{L}$ in the fluid and 0.01 units in the stain. This level if detected in a stain would have been interpreted as being negative for the presence of amylase. The other saliva samples gave strong positive indication of amylase in both the fluids and stains. The average amylase levels for the stains were: 1 day, 0.20 units; 7 days, 0.17 units; 14 days, 0.15 units; 45 days, 0.12 units; and 119 days, 0.08 units. The average amylase level for a saliva stain at 119 days was 4 times greater than the amylase level detected in the 1-day-old seminal stain.

TABLE 3-Amylase activities in saliva stains.

\begin{tabular}{rrrrrrr}
\hline & & \multicolumn{5}{c}{ Levels in Stains $^{b}$} \\
\cline { 2 - 6 } Sample & Fluid Level $^{u}$ & Day 1 & Day 7 & Day 14 & Day 45 & Day 119 \\
\hline 1 & 101 & 0.31 & 0.30 & 0.29 & 0.25 & 0.17 \\
2 & 93 & 0.07 & 0.05 & 0.03 & 0.02 & 0.02 \\
3 & 82 & 0.25 & 0.12 & 0.11 & 0.07 & 0.05 \\
4 & 99 & 0.33 & 0.31 & 0.32 & 0.31 & 0.26 \\
5 & 98 & 0.28 & 0.18 & 0.19 & 0.16 & 0.13 \\
6 & 99 & 0.15 & 0.10 & 0.07 & 0.07 & 0.04 \\
7 & 98 & 0.16 & 0.11 & 0.10 & 0.11 & 0.05 \\
8 & 102 & 0.24 & 0.19 & 0.15 & 0.11 & 0.08 \\
9 & 102 & 0.31 & 0.27 & 0.27 & 0.19 & 0.18 \\
10 & 107 & 0.31 & 0.28 & 0.31 & 0.25 & 0.13 \\
11 & 92 & 0.28 & 0.20 & 0.19 & 0.15 & 0.10 \\
12 & 98 & 0.16 & 0.14 & 0.09 & 0.06 & 0.04 \\
13 & 92 & 0.08 & 0.08 & 0.05 & 0.04 & 0.03 \\
14 & 92 & 0.09 & 0.05 & 0.04 & 0.03 & 0.02 \\
15 & 101 & 0.10 & 0.12 & 0.07 & 0.03 & 0.03 \\
16 & 99 & 0.29 & 0.30 & 0.25 & 0.16 & 0.07 \\
17 & 101 & 0.11 & 0.08 & 0.06 & 0.04 & 0.02 \\
18 & 108 & 0.27 & 0.30 & 0.28 & 0.20 & 0.08 \\
19 & 5 & 0.01 & 0.01 & 0.01 & 0.01 & 0.00 \\
20 & 103 & 0.27 & 0.22 & 0.19 & 0.04 & 0.07 \\
\hline
\end{tabular}

${ }^{a}$ Amylase levels in international units per litre $(\mathrm{U} / \mathrm{L}) \times 10^{3}$.

${ }^{b}$ Amylase levels in international units per litre divided by $5000(\mathrm{U} / \mathrm{L} \div 5000)$. 


\section{Discussion}

High levels of amylase in fluid saliva result in highly detectable levels of amylase in saliva stains. Amylase, being a stable enzyme, is detectable over several months in a saliva stain. The lower levels present in seminal fluid produce little or no detectable amounts of amylase in a stain.

Seminal fluids can have elevated amylase levels, but this is the exception rather than the rule. These levels would be high when compared to normal levels found in semen; however, they would be low when compared to the levels found in saliva. One of the semen samples tested did give a weak positive indication of amylase in its stain, but after several days at room temperature this stain was interpreted as negative for the presence of amylase. The remainder of the semen samples were negative for the presence of anylase in their stains. Saliva stains routinely gave strong positive reactions for the presence of amylase; the one exception was the individual who had an amylase level in the fluid sample of $5000 \mathrm{U} / \mathrm{L}$, and the level detected in a one-day-old stain would have been interpreted as negative for the presence of amylase.

It has been shown that seminal fluids can have elevated amylase levels; however, in most laboratory casework investigators deal with stains and not fluids. These seminal fluids with elevated amylase levels produce amylase levels in stains that are interpreted to be weak positives or negatives. However, fluid saliva, as a rich source of anylase activity, produces highly detectable levels in stains.

There is no absorbance value or color intensity level for the presence of amylase above which the presence of saliva is proven [2]. An absorbance value or color intensity level for amylase, in conjunction with the elapsed time frame between collection and the examination of the stain, can aid in interpreting the possible source of the amylase activity.

In laboratory casework, no amylase activity detected in a stain does not mean that saliva could not be present. It is possible for individuals to have low levels of amylase in their saliva, and as a result a saliva stain from these individuals would lack detectable amylase activity even in a one-day-old stain. In this case, saliva would be present but the results of the amylase testing would indicate that it is not.

When semen could be mixed with saliva and no anylase activity is detected, one cannot rule out the possibility that saliva is present. In such a case, it would be helpful to collect saliva samples from the individuals involved and quantitate the anylase activity present in their saliva.

When a casework stain gives a weak positive reaction for the presence of amylase, the interpretation of its source can be difficult. Amylase activity levels of 0.02 units in stains would be considered weak positives, and the stains could have several possible sources. If semen is also present, the amylase activity could be coming from an individual who has an elevated amylase level in his fluid semen. This could be the case if the stain was reasonably fresh (less than one week old). The amylase activity could be coming from the saliva of an individual who has a low level of amylase activity in his fluid saliva and this low level would be carried over into his saliva stain. This low level of amylase activity could also be due to a normal saliva stain that is several months old.

When there is a possibility of saliva contamination and the level of amylase activity is greater than 0.02 units in a semen-stained area, it is highly unlikely that this amylase activity would be coming from the semen but more likely to have come from saliva if the stain is older than one week. In routine laboratory operations, cases are older than one week before any examinations begin. Salivary amylase levels are high in both fluids and stains, and these levels are detectable over several months. As a result, the time frame between the collection and the examination of evidence is important when drawing conclusions about detectable amylase levels in stains.

\section{Conclusion}

Amylase levels can be elevated in seminal fluid, but this is an exception. Even when the amylase levels in semen are high, these levels are much lower than those found in both fluid saliva 
and saliva stains. Low-level amylase activity in seminal stains from seminal fluids with elevated levels is normally not detectable after one week at room temperature. High amylase levels detected in semen-stained areas in casework may be attributable to saliva when one also considers the time element between collection and examination of the evidence. Low level amylase activity ( 0.02 units or less) in stains does not rule out the possibility that saliva is present. In cases of low level amylase activity in semen-stained areas, the determination of the source of the amylase activity is questionable.

\section{References}

[1] Nelson, D. F. and Kirk, P. L., "The Identification of Saliva," Journal of Forensic Medicine, Vol. 10, No. 1, Jan. 1963, pp. 14-20.

[2] Willott, G. M., "An Improved Test for the Detection of Salivary Amylase in Stains," Journal of the Forensic Science Society, Vol. 14. No. 4, Oct. 1974, pp. 341-346.

[3] Willott, G. M. and Griffiths, "A New Method for Locating Saliva Stains-Spotty Paper for Spotting Spit," Forensic Science International, Vol. 15, No. 1, Jan./Feb. 1980, pp. 79-83.

[4] Baxter, S. J. and Rees, B., "The Identification of Saliva in Stains in Forensic Casework," Medical Science and the Law, Vol. 15. No. 1, Jan. 1975, pp. 37-41.

[5] Kipps, A. E. and Whitehead. P. H., "The Significance of Amylase in Forensic Investigations of Body Fluids," Forensic Science, Vol. 6, No. 3, Dec. 1975, pp. 137-144.

[6] Kipps, A. E., "The Detection of Mixtures of Blood and Other Body Secretions in Stains," Journal of the Forensic Science Society, Vol. 18, No. 3, July 1978, p. 189.

[7] Whitehead, P. H. and Kipps, A. E., "A Testpaper for Detecting Saliva Stains," Journal of the Forensic Science Society, Vol. 15, No. 1, Jan. 1975, pp. 39-42.

[8] Ruston, C., Kipps, A. E., Quarmby, V., and Whitehead, P. H., "The Distribution and Significance of Amylase-Containing Stains on Clothing," Journal of the Forensic Science Society, Vol. 19, No. 1, Jan. 1979. pp. 53-58.

Address requests for reprints or additional information to

Michael J. Auvdel

USACIL-CONUS

P.O. Box L

Forest Park, GA 30051-1386 